\title{
Application of Finite Elements in the Prediction of a Two-Dimensional Temperature Profile for Study of Advection-Conduction Phenomena
}

\author{
Jorge Duarte Forero ${ }^{1 *}$, Guillermo E. Valencia ${ }^{2}$ and Luis G. Obregón ${ }^{3}$ \\ 'Mechanical Engineering Department, DIMER Research Group, Universidad del Atlántico, \\ Barranquilla, Colombia; jorgeduarte@mail.uniatlantico.edu.co \\ ${ }^{2}$ Mechanical Engineering Department, KAI Research Group, Universidad del Atlántico, \\ Barranquilla, Colombia; guillermoevalencia@mail.uniatlantico.edu.co \\ ${ }^{3}$ Chemical Engineering Department, Sustainable Chemical and Biochemical Processes Research Group, \\ Universidad del Atlántico, Barranquilla, Colombia; luisobregon@mail.uniatlantico.edu.co
}

\begin{abstract}
Background/Objectives: Thermodynamic study of advection phenomena relies on the analytical methods to solve a series of Partial Differential Equations (PDE) that generates from multi-dimensional problems, which becomes more and more complex, especially when a 2D or 3D temperature profile is required. Methods: For the solution of heat transfer problems with simultaneous advection-conduction phenomena, finite differences of 2nd, 4th and 6th order were used to approximate the solution of a two-dimensional PDE. Findings: The results show that a low discretization of the system, originated substantial errors in the application of the high order finite, but, when is correctly used, the numeric approximation shows with great precision the temperature profile for the simultaneous advection and conduction heat transfer. Application: To develop a method to accurately predict the temperature profile for complex heat transfer applications where simultaneous advection and conduction are takeninto account.
\end{abstract}

Keywords: Advection, Conduction, Heat Transfer, Numerical Methods, Partial Differential Equations

\section{Introduction}

Advection phenomenaare the transport of a conservative property due to the movement generated by a fluid stream ${ }^{1-4}$; by this definition, convection can be defined as a particular type of advection, which involves the transfer from and a surface and the whole fluid. The properties that are carried with the advected substance are conserved properties such as energy or enthalpy, in which the fluid may be any material that contains thermal energy, such as water or air. Advection is commonly seen in engineering applications that involve moving fluids ${ }^{5}$, such as meteorology and physical oceanography, where the transport of some property of the atmosphere or ocean, such as heat, humidity, salinity or pollutants, are of special concern (due to the environmental effects these variables can generate).

Thermodynamic study of advection phenomena relies on the analytical methods to solve a series of Partial Differential Equations (PDE) that generates from multidimensional problems ${ }^{6}$. Besides, given the fact advection occurs with another heat transfer mechanisms, such as conduction (which is defined as a $2^{\text {nd }}$ order differential equation $^{7}$ ), the analytical solution becomes more and more complex, particularly when a $2 \mathrm{D}$ or $3 \mathrm{D}$ temperature profile is required. Because of that, it is necessary to find an alternative method that Equation (1) ensures accuracy 
in the results, Equation (2) can be easier to solve, and Equation (3) can be programmed in commercial software.

From the main aspects previously shown, the most suitable option is the application of numerical methods in the solution of PDE, which applies a discretization of these, in order to reduce them to algebraic expressions, which are way easier to handle that their differential counterparts ${ }^{8}$; example of their usefulness, resides in a huge quantity of investigations done with the help of numerical methods to solve even 3D, non-steady $\mathrm{PDE}^{9-13}$. From this, the objective of the present work, is the solution of heat transfer problems, with simultaneous advectionconduction phenomena, using finite differences of $2^{\text {nd }}, 4^{\text {th }}$ and $6^{\text {th }}$ order to approximate the solution of an advectionconduction two-dimensional PDE, using commercial software $\left(\mathrm{MATLAB}^{\circ}\right)$ to do the calculations required. The results were evaluated to different values of discretization, to establish a comparison between the analytic solution and the numerical method used.

\section{Methodology}

This section presents a detailed description of the general purpose of the study, and the fundamental equations implemented for the realization of the investigation.

\subsection{The General Aim of the Study}

The main purpose of the study developed is to apply numerical methods in the solution of two-dimensional PDE (Partial Differential Equations), and their application to predict the temperature profile near a hot spot, (such as a welding point or a heater in an enclosed space), by using finite differences to approximate the solution of advection-conduction equation over a previously defined domain. To analyze the degree of exactitude from the numerical method used, 2nd order, 4th order and 6th order finite differences approximations were programmed using the commercial package MATLAB ${ }^{\circledR}$ and applied to the case ${ }^{8}$; to verify the method accuracy, the deviation from analytical solution was obtained, taking in account the simultaneous presence of advection and conduction phenomena, over a gas (air, in this case).

\subsection{Fundamental Equations}

In a general form, the advection equation, applied to a case where conduction occurs simultaneously, can be defined as the Equation (1),

$$
\frac{\partial T}{\partial t}+a \cdot \frac{\partial T}{\partial x}=v \cdot \frac{\partial^{2} T}{\partial y^{2}}
$$

Where $a$ is the velocity of the stream and $v$ the kinematic viscosity of the fluid. From the Equation (1) and taking into account a space enclosed by a length $L$ in both $x$ and $y$ directions, a solution can be found using the following initial boundaries:

$$
\begin{aligned}
& \frac{T}{T_{h s}}(x, y, 0)=1 \text { for } 0<x<L, 0<y<L \\
& \frac{T}{T_{h s}}(x, 0, t)=0 \text { for } 0<x<L, t \geq 0 \\
& T(0, y, t)=T_{h s} \text { for } 0<y<L, t \geq 0
\end{aligned}
$$

Where $T_{\mathrm{hs}}$ is the temperature of the hot spot (the flame), which is related to y position with the expression

$$
T_{h s}=\sin \left(\frac{\pi \cdot y}{L}\right)
$$

With Equations (2) to (4) as boundary conditions, and (5) as the initial condition, the solution methods for the PDE (1) allow to calculate the temperature profile in an analytic form, shown in the Equation (6) for steady state (where the time derivative becomes zero):

$$
T(x, y)=\sin \left(\frac{\pi y}{L}\right) \cdot \exp \left(\frac{\pi^{2} v}{\rho C_{p} L^{2}} \cdot x\right)
$$

To solve the same PDE in a numerical form, a set of finite numerical differences was applied to approximate the first and second derivatives required; since these differences are truncated expansions of the Taylor series ${ }^{6}$, these usually come with an inherent error added. Using forward finite differences, the first and second derivatives of a function $\mathrm{f}(\mathrm{x})$ can be calculated using the Equations (7) and (8),

$$
\begin{aligned}
& \beta f_{i-2}+\alpha f_{i-1}+f_{i^{\prime}}+\alpha f_{i+1}+\beta f_{i+2} \\
= & c \frac{f_{i+3}-f_{i-3}}{6 h}+b \frac{f_{i+2}-f_{i-2}}{4 h}+a \frac{f_{i+1}-f_{i-1}}{2 h} \\
\beta f_{i-2^{2}} & +\alpha f_{i-1^{-}}+f_{i^{\prime \prime}}+\alpha f_{i+1}+\beta f_{i+2^{2}}=c \frac{f_{i+3}-2 f_{i}+f_{i-3}}{9 h^{2}} \\
& +b \frac{f_{i+2}-2 f_{i}+f_{i-2}}{4 h^{2}}+a \frac{f_{i+1}-2 f_{i}+f_{i-1}}{h^{2}}
\end{aligned}
$$

Where $\alpha, \beta, a, b$ and $c$ are specific coefficients, which are dependent of the error order used in the approximation, 
calculated from the Taylor series expansion ${ }^{14,15}$, these values were calculated by previous authors, and are shown in Table 1 and 2.

Table 1. First derivative coefficients for finite differences

\begin{tabular}{|l|l|l|l|l|l|}
\hline Error Order & $\alpha$ & $\beta$ & a & b & c \\
\hline 2 & 0 & 0 & 1 & 0 & 0 \\
\hline 4 & $1 / 4$ & 0 & $3 / 2$ & 0 & 0 \\
\hline 6 & $1 / 3$ & 0 & $14 / 9$ & $1 / 9$ & 0 \\
\hline
\end{tabular}

Table 2. Second derivative coefficients for finite differences

\begin{tabular}{|l|l|l|l|l|l|}
\hline Error Order & $\alpha$ & $\beta$ & $\mathbf{a}$ & $\mathbf{b}$ & $\mathbf{c}$ \\
\hline 2 & 0 & 0 & 1 & 0 & 0 \\
\hline 4 & $1 / 10$ & 0 & $6 / 5$ & 0 & 0 \\
\hline 6 & $2 / 11$ & 0 & $12 / 11$ & $3 / 11$ & 0 \\
\hline
\end{tabular}

\section{Result and Discussion}

The objective of the study was to use finite differences to approximate the temperature profile for a case of twodimensional heat transfer taking in account simultaneous conduction and advection, which is a common situation in some engineering applications that involve a fluid. To do this, a boundary of length $L=1 \mathrm{~m}$ was defined for both $x$ and $y$ directions, and the temperature profile for slowmoving atmospheric air $(\approx 0.1 \mathrm{~m} / \mathrm{s})$ from a heat source at $60^{\circ} \mathrm{C}$ was determined using polynomials with a different order.

\subsection{Comparison of the Finite Differences Method Used}

For comparison purposes, the analytic solution of the advection-conduction PDE (1) in steady state was calculated, as shown in Figure 1. From this figure, it is shown that the temperature decreases slower in the $\mathrm{x}$-direction, due to the fact advection takes place along that axis, as is suggested in the Equation (1); in this case, the low velocity of the air stream that moves near the heat source, induces 7 the thermal equilibrium between it and the air, which creates a profile with a low slope, in which the gas exits the boundary with approximately $50 \%$ of the source temperature.

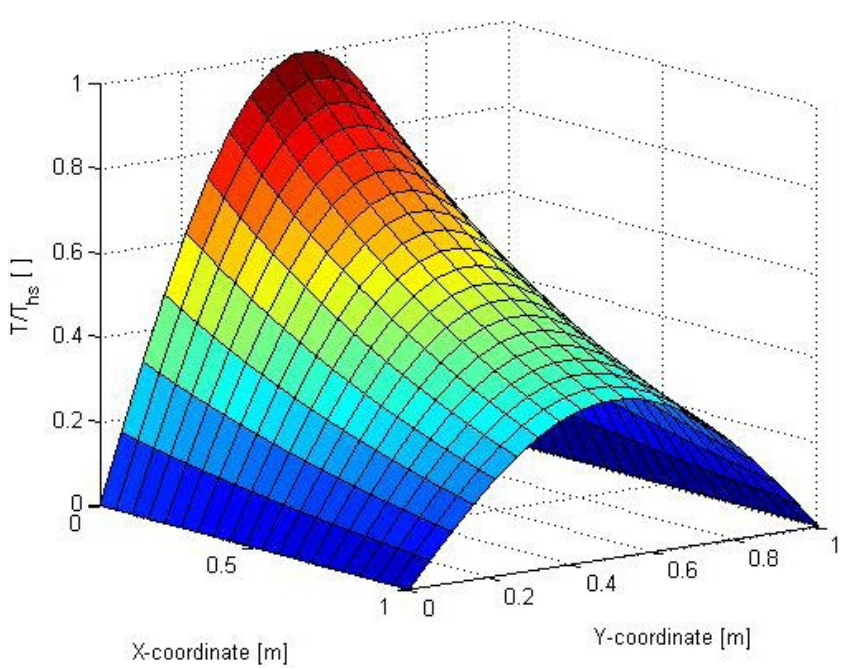

Figure 1. Analyticalsolutionof PDE (1).

For the numerical method application, polynomials of a different order (as said before), were formulated from Equations (7) and (8), together with the coefficients shown in Tables 1 and 2. The application of the finite differences equations required the discretization of the boundary length in a mesh of $(x, y)$ coordinates, and three cases were evaluated: a $20 \times 20$ mesh (that is, 20 divisions in $\mathrm{x}$-direction and $\mathrm{y}$-direction), a 60x60 mesh, and an 80x80 mesh; the results for each mesh type are shown in Figures 2 to 4 . To establish a method to compare the accuracy of each solution, the base-10 logarithm of the absolute error was calculated between the model and analytical solution, for the temperature profile in the symmetry axis along the $\mathrm{x}$-axis, as shown in Figures 5 to 7.

From these figures, it is evident that the low discretization generated with the $20 \times 20$ mesh originated substantial errors in the application of the $4^{\text {th }}$ and $6^{\text {th }}$ order polynomials, but at higher values of the mesh, the error is considerably lower. As the error estimated with the highest discretization used, falls under a range of $10^{-8}$ to $10^{-12}$, it is evident that the numeric approximation of the Equation (6) shows with great precision the temperature profile for the simultaneous advection and conduction heat transfer. However, given the fact that a more discretized mesh implies more elements, a way to reduce the computational load and at the same time obtain a robust approximation from the profile, is using the $2^{\text {nd }}$ order polynomial with a low mesh (20x20). 


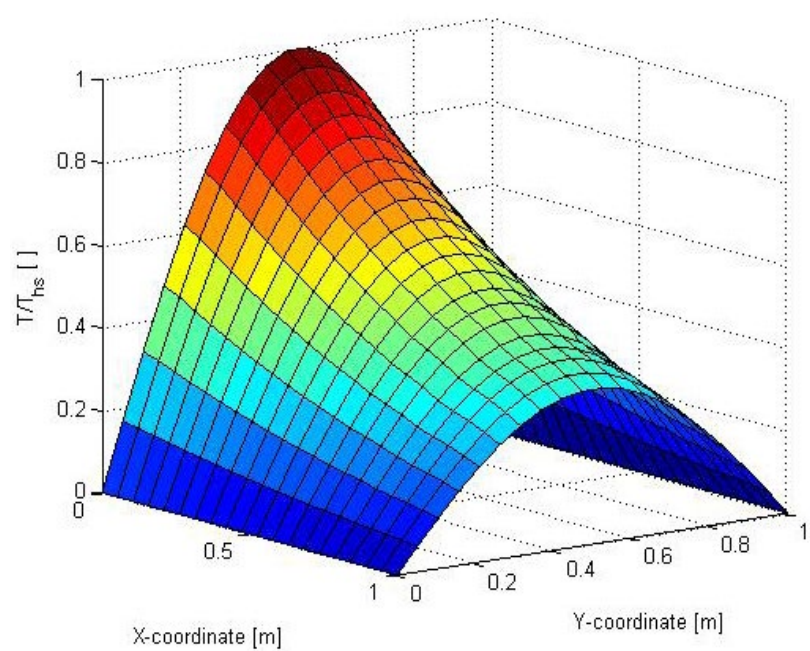

Figure 2. Numerical solution of PDE (1) with a 20x20 mesh.

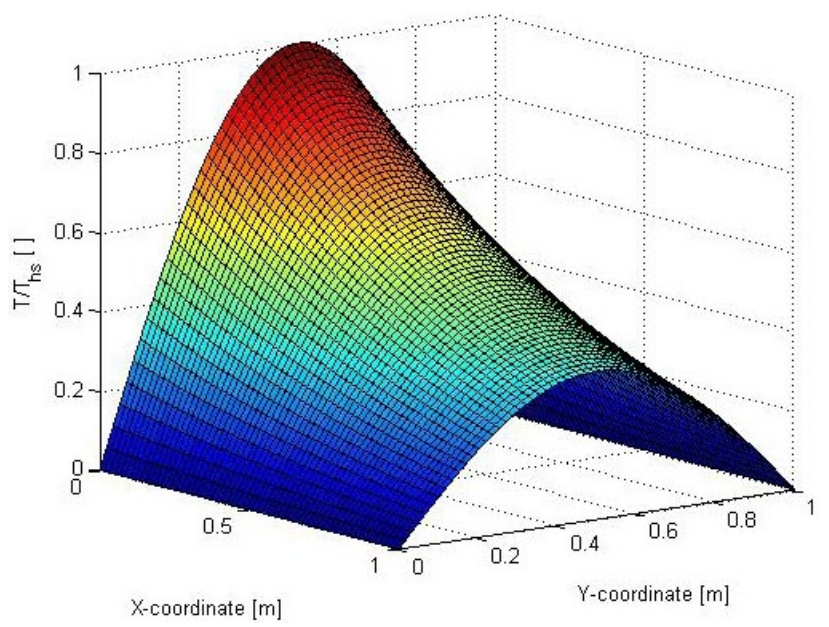

Figure 3. Numerical solution of PDE (1) with a $60 \times 60$ mesh.

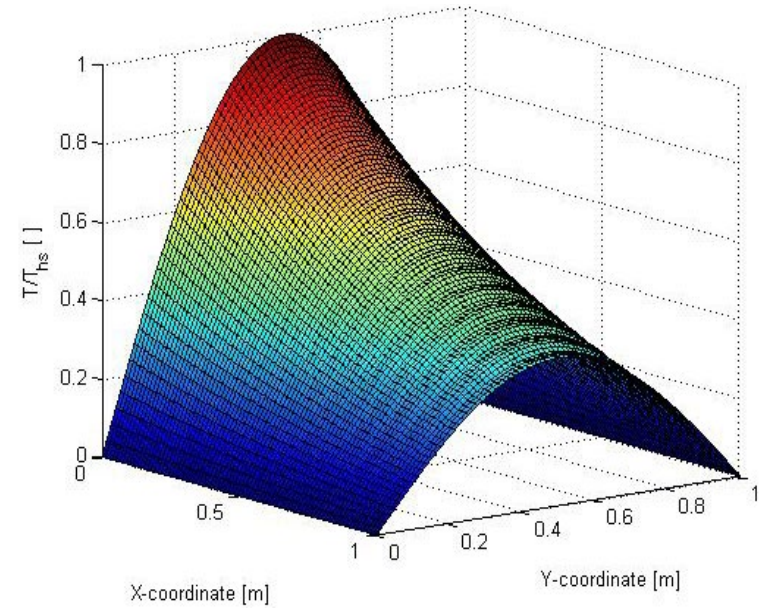

Figure 4. Numerical solution of PDE (1), with a 80x80 mesh.

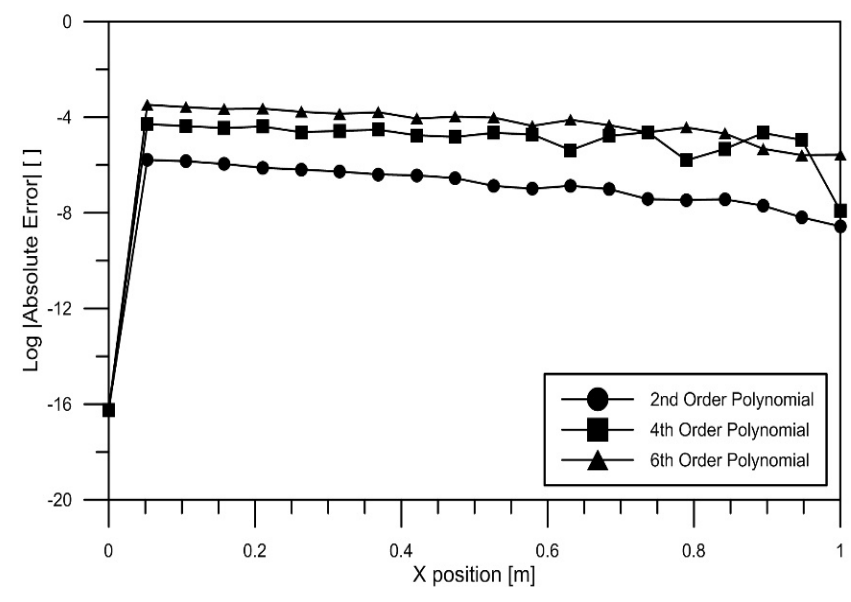

Figure 5. Error calculated with the polynomials used, for a 20x20 mesh.

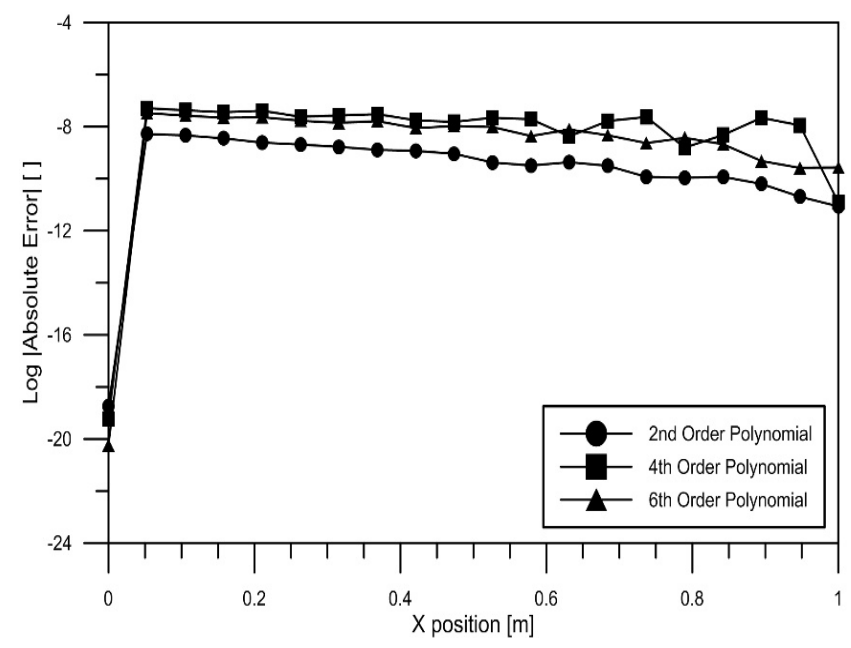

Figure 6. Error calculated with the polynomials used, for a 60x60 mesh.

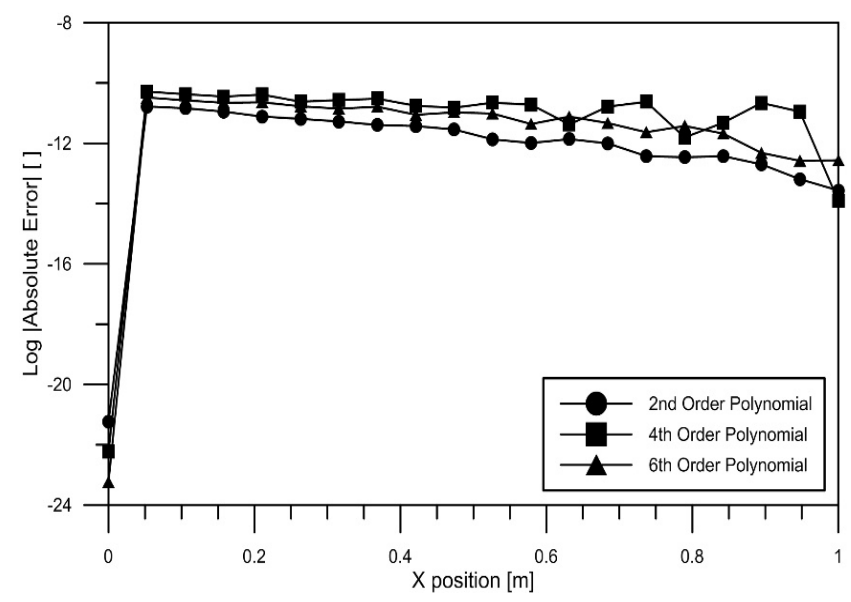

Figure 7. Error calculated with the polynomials used, for a80x80 mesh. 


\section{Conclusions}

As a conclusion, the investigation demonstrated the efectiveness of the numerical method in the solution of Partial Differential Equations, with a method easier to formulate, program and solve than the analytic solution, which represents a time and cost saving; besides that, the same method allows to obtain a variable precision in the results, by selecting the discretization level and polynomial order most suitable to the available computational load, varying from a robust approximation (with $2^{\text {nd }}$ order polynomials with a $20 \times 20$ mesh) to the highest degree of approximation, using a high discretization $\left(6^{\text {th }}\right.$ order polynomials with an $80 \times 80$ mesh). This fact makes the application of this method suitable for the development of software packages that automatize the calculation and solution algorithm, to create a useful tool in the solution of $2 \mathrm{D}$ heat transfer problems.

\section{References}

1. Molina-Giraldo N, Blum P, Zhu K, Bayer P, Fang Z. A moving finite line source model to simulate borehole heat exchangers with groundwater advection. International Journal of Thermal Sciences. 2011; 50(12):2506-13. Crossref

2. Li H, Nagano K, Lai Y. Heat transfer of a horizontal spiral heat exchanger under groundwater advection. International Journal of Heat and Mass Transfer. 2012; 55(23-24):681931. Crossref

3. Caissie D, Luce CH. Quantifying streambed advection and conduction heat fluxes. Water Resources Research. 2017; 53(2):1595-624. Crossref

4. Asgari M, Akhlaghi M. Transient thermal stresses in twodimensional functionally graded thick hollow cylinder with finite length. Archive of Applied Mechanics. 2010; 80(4):353-76. Crossref

5. Sawyer AH, Cardenas MB, Buttles J. Hyporheic temperature dynamics and heat exchange near channel-spanning logs. Water Resources Research. 2012; 48(1):1-11. Crossref

6. Kreyszig H. Advanced engineering mathematics. 19th ed. USA: John Wiley and Sons, Inc. 2011. p. 1-1244.

7. Incropera FP, Dewitt DP, Bergman TL, Lavine AS. Fundamentals of heat and mass transfer. $12 \mathrm{ed}$. Hoboken NJ: John Wiley and Sons, Inc. 2007. p. 1-9997.

8. Cogne C, Labouret S, Peczalski R, Louisnard O, Baillon F, Espitalier F. Theoretical model of ice nucleation induced by acoustic cavitation. Part 1: Pressure and temperature profiles around a single bubble. Ultrasonics Sonochemistry. 2016; 29:447-54. Crossref PMid:26044460

9. Rana P, Bhargava R. Flow and heat transfer of a nanofluid over a nonlinearly stretching sheet: A numerical study. Communications in Nonlinear Science and Numerical Simulation. 2012; 17(1):212-26. Crossref

10. Raymond J, Therrien R, Gosselin L, Lefebvre R. Numerical analysis of thermal response tests with a groundwater flow and heat transfer model. Renewable Energy. 2011; 36(1):315-24. Crossref

11. Soleimani S, Sheikholeslami M, Ganji DD, Gorji-Bandpay M. Natural convection heat transfer in a nanofluid filled semi-annulus enclosure. International Communications in Heat and Mass Transfer. 2012; 39(4):565-74. Crossref

12. Zhao J. Compact finite difference methods for high order integro-differential equations. Applied Mathematics and Computation. 2013; 221:66-78. Crossref

13. Lele SK. Compact finite difference schemes with spectrallike resolution. Journal of Computational Physics. 1992; 103(1):16-42. Crossref

14. Camargo C, Garcia C, Forero JED, Rincon A. Modelo estadístico para la caracterizacion y optimizacion en bombas perifericas. Ingenieria y Desarrollo. 2017; 36(1):18-39. Crossref 